\title{
Quelques principes simples pour gérer les crises complexes
}

Jean-Pierre Chevènement

\section{(2) OpenEdition}

1 Journals

Édition électronique

URL : http://journals.openedition.org/communicationorganisation/2280

DOI : 10.4000/communicationorganisation.2280

ISSN : $1775-3546$

Éditeur

Presses universitaires de Bordeaux

Édition imprimée

Date de publication : 1 novembre 1999

ISSN : 1168-5549

Référence électronique

Jean-Pierre Chevènement, "Quelques principes simples pour gérer les crises complexes »,

Communication et organisation [En ligne], 16 | 1999, mis en ligne le 27 mars 2012, consulté le 19 avril

2019. URL : http://journals.openedition.org/communicationorganisation/2280 ; DOI : 10.4000/

communicationorganisation. 2280

Ce document a été généré automatiquement le 19 avril 2019.

(c) Presses universitaires de Bordeaux 


\title{
Quelques principes simples pour gérer les crises complexes
}

\author{
Jean-Pierre Chevènement
}

\section{NOTE DE L'ÉDITEUR}

Article publié dans le $\mathrm{n}^{\circ} 283$ de la revue mensuelle de l'ENA.

1 Les risques provenant des techniques ou de la nature se présentent de façon toujours complexe et mettent en jeu de nombreux acteurs. Leur gestion, la résolution et les crises qu'ils engendrent ne peuvent que s'appuyer sur des principes simples.

2 Les crises résultant des risques technologiques et naturels présentent une caractéristique propre : à la différence des autres, elles ne consistent pas à lutter contre un ennemi ou une agression, mais à faire face à des événements qui naguère, s'apparentaient, soit au cas de force majeure, soit la «nature des choses ». Elles impliquent par ailleurs un nombre et une diversité d'acteurs bien supérieurs à ceux que l'on peut trouver dans les autres domaines opérationnels du ministère de l'intérieur. Leur résolution implique donc, à la fois de faire appel aux principes communs à tous les types de crise et aussi d'offrir des réponses spécifiques : il faut fédérer l'action de partenaires aux motivations diverses et coordonner leurs actions.

Une crise se caractérise par la désorganisation des structures sociales et les catastrophes technologiques ou naturelles sont bien de nature à bouleverser les dispositifs habituels.

4 La qualité du dispositif existant en France, et sans doute une part de chance, ont permis dans les dernières années que les cataclysmes ne dégénèrent pas en crise. Mais des exemples récents chez nos voisins rappellent que la menace est bien réelle. 


\section{Les facteurs de complexité}

5 Les accidents majeurs ne connaissent pas les limites institutionnelles. C'est un élément de leur complexité. Les conséquences de ces accidents ne peuvent être traitées au niveau d'une seule commune, voire au niveau d'un seul département. Dans nombre de cas, elles traversent même les frontières nationales.

6 La variété des risques est telle, que la réponse ne peut être univoque. Ainsi, en ce qui concerne les risques technologiques, la maitrise du danger que crée l'existence d'une installation chimique relève d'une méthodologie différente de celle qui permettra de réduire celui qui résulte du transport des matières dangereuses. Les installations nucléaires de base, caractérisées par une relative homogénéité au plan national, doivent être traitées selon un processus encore différent.

7 Certains risques naturels, par leur récurrence, ont conduit à mettre en place un dispositif spécifique, les grands incendies de forêt en sont l'exemple le plus achevé. Moyens aériens anti-feu de nouvelle génération, centre coordination opérationnelle de Valabre (13), unités militaires spécialisées, symbolisent l'intervention désormais bien organisée de l'État. Et l'on peut aussi constater combien la campagne annuelle contre les feux de forêt à modelé les moyens et les façons de travailler des services départementaux d'incendie et de secours.

8 Il existe un troisième type de risques, moins bien connu que les deux précédents, celui lié aux systèmes de transport, qu'ils soient d'énergie ou d'information. II est de notre responsabilité, en liaison avec les organismes compétents, de préparer des réactions planifiées à des ruptures majeures des réseaux de télécommunications ou de données informatisées. Ceci suppose l'acquisition de nouvelles compétences au titre de la défense civile. La création de la Direction de la défense et de la sécurité civiles, issue de la fusion du service du haut fonctionnaire de défense et de l'ex-direction de la sécurité civile en est une première illustration.

\section{L'unité de décision est fondamentale}

Situations complexes encore, du fait de la multiplicité des intervenants : il s'agit, non seulement des services publics, mais aussi des créateurs de risque et enfin et peut-être surtout, de la population et des médias.

10 En ce qui concerne les services publics, le maire, au titre du code des communes, et selon une rédaction qui date maintenant de 1884, est responsable de la sécurité des citoyens. Mais dès qu'il s'agit d'un événement majeur, le niveau communal ne peut, ni ne doit, réagir isolément. Ainsi, le dispositif français repose-t-il sur le rôle de coordonnateur du préfet. Sur un plan concret, l'unité de décision est fondamentale. Les textes, au premier plan desquels se trouve la loi de 1987 sur la sécurité civile, fixent cette place, et celle du ministère de l'Intérieur à l'échelon national. Le préfet devra donc encadrer les actions municipales, voire se substituer à celles-ci lorsqu'elles sont submergées, au moins dans un premier temps, par la catastrophe.

11 Véritable meneur de jeu, le préfet s'appuie sur la collaboration des services de l'État et des sapeurs-pompiers. Il réunit, pour maitriser la crise, non seulement des services d'urgence habitués à l'action immédiate, mais aussi ceux qui, chargés au quotidien de 
conduire l'action générale, se retrouvent lors de la catastrophe, confrontés à des modes de fonctionnement totalement dégradés et auxquels ils ne sont pas totalement préparés. Les catastrophes les plus récentes ont montré de plus que les services publics, tels que EDF et France Télécom, sont des acteurs majeurs pour le rétablissement d'une situation. Le préfet doit alors s'appuyer sur des Organismes qui, en fonctionnement normal, veulent se rapprocher toujours plus de la logique propre aux entreprises commerciales, et qui, sans transition, retrouvent leur première destination de service public, au service de sinistrés et non plus de clients.

12 Face à une désorganisation majeure, les seules forces des services publics ne pourront suffire : la France a la chance de pouvoir disposer d'un ensemble de bénévoles organisés en associations : Fédération nationale de protection civile, Croix rouge, radioamateurs, pour ne citer que les plus connues, sont en mesure d'apporter une contribution efficace dès lors qu'elles sont pleinement intégrées au dispositif, en cohérence avec l'action des services.

La diversité des phases d'une crise, de la prévention au retour à la normale implique de plus, que l'action des services publics, coordonnée sur le terrain par le préfet, soit organisée et soutenue au niveau national par un grand nombre de ministères. Ceux-ci, d'ailleurs, s'appuient bien souvent sur des établissements publics, dont la nécessaire autonomie ne doit pas conduire à un éclatement de l'action de l'État, alors que c'est vers ce dernier, qu'en situation de catastrophe, se dirigent toutes les exigences. est mal identifiable pour les événements d'origine naturelle, elle est d'un poids particulier lors d'accidents technologiques. Dans ce cas, il faut rappeler que, lorsque le phénomène est limité à l'intérieur d'une installation, c'est l'exploitant de celle-ci qui doit apporter la première réponse selon les modalités fixées par des réglementations comme celle des installations classées. Toute la difficulté est alors d'anticiper l'extension du sinistre et ses conséquences possibles sur la population. Logique d'entreprise et sécurité du public doivent se retrouver pour une action commune.

La troisième famille d'acteurs (la population et les médias) est la plus importante puisque toute notre action est tournée vers le citoyen, et les différents acteurs doivent se mettre au service de sa sécurité.

De l'information préventive à l'assistance après la catastrophe, en passant par une prise en charge complète des victimes, pendant la phase d'urgence, il faut alors trouver les solutions pour agir. Il faut savoir témoigner aux sinistrés un soutien chaleureux, personnalisé et efficace et simultanément, faire passer à l'opinion nationale, voire internationale l'information nécessaire. Les associations d'usagers ou de sinistrés doivent alors être considérées comme des collaborateurs véritables de l'action des pouvoirs publics.

17 Les médias ont pu être considérés par le passé comme des perturbateurs, mais l'on sait aujourd'hui que leur action peut être un des moyens pour qualifier l'événement et diminuer l'irrationnel. La aussi, leurs attentes, leur dynamique et même leurs exigences, doivent être intégrées dès le stade de la préparation.

Enfin, il faut rappeler que dans cet ensemble d'interactions, l'exigence est de plus en plus forte. Le citoyen se tourne désormais vers les pouvoirs publics et leur impute une obligation de résultats. 

chargées d'apporter les solutions. Il ne s'agit plus de responsabilité collective, autrement dit, administrative, mais de responsabilité personnelle, et donc pénale. Or, si celle-ci a toujours existé, elle est aujourd'hui, d'une façon qui peut paraître parfois brutale, mise en œuvre effectivement. La justice est alors, à la fois, partenaire dans la résolution de la crise et censeur de l'action.

\section{Répondre par la coordination}

La complexité est donc une donnée. Faire qu'elle ne soit pas une contrainte, mais une richesse par sa potentialité en synergies, suppose une coordination des plus développées. Pour ce faire, il faut se comprendre, connaître les moyens dont on peut disposer, savoir comment les employer, quand le faire, et enfin, reconnaitre la place du commandement, sans oublier la nécessité de faire savoir.

Pour pouvoir se coordonner, il faut d'abord parler un même langage. Autrement dit, tous les acteurs doivent pouvoir partager une culture commune de crise. Les administrations centrales et en tout cas la direction de la défense et de la sécurité civile, élaborent en ce sens une doctrine, non pas intellectuelle, mais qui résulte des leçons données par la réalité. C'est l'exploitation de celles-ci qu'est le retour d'expérience et sa diffusion qui permettront progressivement de donner, non pas des régies immuables ou des recettes, mais des points de repère.

Dans l'action, la coordination dépend d'abord d'une bonne circulation de l'information : rendre compte sans délai, informer en continu les autres acteurs, rechercher inlassablement le renseignement, l'exploiter, doivent être de véritables obsessions pour les équipes de crise. Face au choc qui résulte des catastrophes, lorsque l'information est absente, confuse ou parfois aussi trop abondante, il est de leur responsabilité d'approcher le plus exactement possible la réalité, alors que celle-ci évolue à grande vitesse. Ceci ne sera possible que par la réactivité et l'anticipation, donc par le recours a des structures de coordination prédéfinies et entrainées correctement. La mise en place de PC opérationnels proches du sinistre, le soutien d'un PC fixe, le Centre opérationnel de défense, installé en préfecture, sont des solutions éprouvées. Il convient de les systématiser et de les doter de moyens indispensables. Ces centres coordonnent des cellules de crise satellites aux missions plus précises.

Ces cellules de crise ne peuvent fonctionner que si elles s'appuient sur des plans de secours, eux-mêmes rondes sur une analyse du risque. C'est dans cette logique que sont mis en place différents outils d'analyse du risque, dont le schéma départemental d'analyse et de couverture des risques (SDACR) est un exemple. Celui-ci a pour but d'assurer la cohérence entre l'existence du risque, sa connaissance et la réponse opérationnelle tout spécialement par les moyens des sapeurs-pompiers. Les études de danger sur certaines installations industrielles procèdent de la même logique.

Coordonner, c'est aussi pouvoir concentrer les moyens. Ceux-ci peuvent venir des collectivités territoriales, des entreprises, des services d'État dans le département ou des départements voisins. Il peut s'agir aussi de moyens nationaux. La direction de la défense et de la sécurité civiles dispose ainsi d'unités d'instruction et d'intervention de la sécurité civile (UIISC) projetables en France comme à l'étranger sur des catastrophes de toute nature, de moyens aériens (avions et hélicoptères), d'équipes de démineurs mais aussi 
d'équipes d'appui mises a la disposition du préfet lorsque celui-ci souhaite renforcer son état-major.

D'autres ministères et des établissements publics peuvent également apporter une contribution efficace : la Défense, mais aussi dans le domaine plus spécialisé des plans de secours, l'Équipement, l'Environnement, le Commissariat à l'énergie atomique (CEA) ou encore l'office de protection des rayonnements ionisants (Opri).

L'effort de coordination ne peut avoir de résultat positif que s'il intègre la communication. À la différence d'autres crises, où le secret peut être une condition de réussite, l'expérience montre que pour les risques naturels et technologiques, la diffusion vers les médias en particulier est un facteur de succès. Aujourd'hui encore, les responsables considèrent souvent que tous leurs efforts doivent être consacrés à porter secours et à limiter les conséquences de la catastrophe et que le temps consacré à la communication ne peut être pris au détriment de cette mission fondamentale. Pourtant, qu'il s'agisse de transmettre l'alerte ou de faire le point sur les réponses apportées pour revenir à une situation normale, la contribution des médias est déterminante. À l'inverse, le silence des responsables sera interprété comme une volonté de préserver un secret, même imaginaire. La polémique infondée, la diffusion des rumeurs les plus absurdes, deviennent alors des facteurs aggravants qui peuvent faire à eux seuls d'une situation objectivement maîtrisée, une crise subjective et subie.

Multiplicité des partenaires, multiplicité des objectifs de la coordination, ne doivent pas faire oublier que face à la désorganisation, il faut décider. Dès lors que le préfet a su associer tous les partenaires concernés, prendre leur avis et s'être fait une idée exacte de la situation, c'est bien à lui qu'appartient, au plan local, la décision.

Répondre efficacement aux catastrophes naturelles ou technologiques suppose donc une profonde connaissance du rôle de chacun. Celle-ci débouche alors sur un respect mutuel et des rapports de confiance. Ce type de crise est caractérisé par un besoin d'ouverture, d'autant plus difficile que le premier réflexe face à un ébranlement brutal est souvent le repli sur soi-même de chaque institution, mais aussi de chaque individu. Les principes qui se mettent en place aujourd'hui ne se fondent alors plus seulement sur une autorité décrétée, mais un besoin essentiel de cohérence au service du citoyen.

\section{RÉSUMÉS}

Les risques majeurs originaires des techniques ou de la nature sont toujours complexes: ils mettent en jeu de nombreux acteurs. Les réactions aux risques, leur gestion et la résolution des crises qu'ils peuvent provoquer exigent de respecter des principes simples. Indépendamment des responsabilités engagées les actions mises en place visent à motiver, fédérer, unir et coordonner les partenaires.

The serious risks induced either by technics or nature itself are always complex : they imply many actors. The reactions to those risks, the handling of these, and the solving of the crises they can trigger require a strict commitment to certain plain rules. Notwithstanding the 
responsabilities engaged, the steps that have been taken tend to modify, federate, unify an coordinate the partners.

\section{AUTEUR}

\section{JEAN-PIERRE CHEVÈNEMENT}

ENA Promotion Stendhal 1965. Né en 1939 à Belfort dont il est l'élu depuis 1973 (député maire), Jean-Pierre Chevènement a été aux côtés de François Mitterrand l'un des artisans de la refondation du parti socialiste en 1971. De 1981 à ce jour, il a occupé plusieurs postes ministériels importants : Recherche Industrie, Éducation Nationale, Défense et Intérieur. Deux idées directrices : le rôle de l'État et la souveraineté nationale. 